Chirurg 2013 $\cdot 84: 598$

DOI 10.1007/s00104-013-2545-5

Online publiziert: 13. Juni 2013

(c) Springer-Verlag Berlin Heidelberg 2013
H. Dralle ·J. Hein

Universitätsklinik für Allgemein-, Viszeral- und Gefäßchirurgie,

Medizinische Fakultät, Universitätsklinikum Halle, Halle (Saale)

\section{Parathyreoidektomie bei unklarer Lokalisation}

\section{Minimal-invasiv oder offen}

\section{Originalpublikation}

Nehs MA, Ruan DT, Gawande AA et al (2013) Bilateral neck exploration decreases operative time compared to minimally invasive parathyroidectomy in patients with discordant imaging. World J Surg. DOI 10.1007/s00268013-2007-8

\section{Hintergrund und Fragestellung}

Nebenschilddrüsenoperationen gehören nach Schilddrüsenoperationen $\mathrm{zu}$ den häufigsten Eingriffen am endokrinen System. In den USA werden jährlich ungefähr 100.000 Patienten wegen eines primären Hyperparathyreoidismus (pHPT) operiert. Etwa 90\% der Patienten mit pHPT haben eine sog. Eindrüsenerkrankung (solitäres Adenom [NSDA]), $10 \%$ eine Mehrdrüsenerkrankung (Doppeladenom oder noduläre Mehrdrüsenhyperplasie). Zahlreiche Untersuchungen haben gezeigt, dass bei konkordanter Bildgebung durch Sonographie und MIBI (Methoxy-isobutyl-isonitril)-Szintigraphie minimal-invasiv fokussierte Parathyreoidektomien mit vergleichbarem Erfolg wie konventionell-offene Parathyreoidektomien mit bilateraler Halsexploration durchgeführt werden können. Die in der vorliegenden Arbeit analysierte Frage war daher, ob auch bei dyskordanter Bildgebung oder fehlender präoperativer Lokalisation das minimal-invasive Vorgehen gegenüber der konventionell-offenen bilateralen Exploration von Vorteil ist.

\section{Methoden}

Unter Verwendung eines prospektiv geführten Datenprotokolls wurden zwi- schen 2005 und 2009324 Nebenschilddrüsen(NSD)-Explorationen durchgeführt. Bei 76 Patienten lag präoperativ eine dyskordante Bildgebung vor, d. h. Ultraschall und MIBI-Szintigraphie lokalisierten das gesuchte NSDA auf unterschiedlichen Halsseiten oder das NSDA wurde nur durch eines der beiden Lokalisationsverfahren darstellt.

\section{Ergebnisse}

Bei 62 der 76 Patienten erfolgte eine bilaterale Halsexploration ohne intraoperative Parathormon (IOPTH)-Bestimmung, bei 14 eine unilaterale Exploration mit IOPTH. Die Heilungsraten beider Operationsverfahren waren vergleichbar (100\% vs. $98 \%$; $\mathrm{p}=1,0)$. Bei 4 der $14 \mathrm{~Pa}-$ tienten mit unilateraler Exploration wurde wegen eines inadäquaten IOPTH-Abfalls $(<50 \%)$ zur bilateral-offenen Exploration konvertiert und in 2 der 4 Fälle ein kontralaterales Doppeladenom nachgewiesen. Die mediane Operationsdauer der unilateralen Exploration mit IOPTH-Bestimmung betrug $96 \mathrm{~min}$, die der bilateralen Exploration ohne IOPTH-Bestimmung 52 min ( $\mathrm{p}=0,0027)$. Die Rate von Mehrdrüsenerkrankungen betrug bei den 76 Fällen $30 \%$.

\section{Diskussion und Fazit}

Die Autoren teilen nicht mit, warum im individuellen Fall bei präoperativ dyskordanter Bildgebung eine uni- oder bilaterale Exploration erfolgte. Die Ergebnisse lassen jedoch folgende Schlussfolgerungen zu:
- Eine präoperativ dyskordante Bildgebung (gleiches gilt für den pHPT mit fehlendem Lokalisationsnachweis) bedeutet ein erhöhtes Risiko für das Vorliegen einer Mehrdrüsenerkrankung. In dieser Situation ist eine bilaterale Exploration ohne IOPTH nicht nur der unilateralen Exploration mit IOPTH gleichwertig, sondern in doppelter Hinsicht kostengünstiger: Sie spart die Kosten des IOPTH und sie spart Zeit (die Zeit vor allem, die die IOPTH-Bestimmung braucht, in der vorliegenden Studie ca. 45 min).

- Beim unilateralen Vorgehen sollte auf eine IOPTH-Bestimmung nicht verzichtet werden, um kontralaterale Adenome oder Mehrdrüsenhyperplasien nicht zu übersehen. Mehrdrüsenerkrankungen sind der Hauptrisikofaktor für eine Persistenz des pHPT [1], sodass bei unilateraler Exploration und inadäquatem PTH-Abfall in aller Regel eine bilaterale Exploration erforderlich ist.

\section{Korrespondenzadresse}

Prof. Dr. Dr. h.c. H. Dralle

Universitätsklinik für Allgemein-, Viszeral- und Gefäßchirurgie, Medizinische Fakultät, Universitätsklinikum Halle, Ernst-Grube-Str. 40, 06097 Halle (Saale) henning.dralle@uk-halle.de

Interessenkonflikt. Keine Angaben

\section{Literatur}

1. Schneider DF, Mazeh H, Sippel RS, Chen H (2012) Is minimally invasive parathyroidectomy associated with greater recurrence compared to bilateral exploration? Analysis of more than 1,000 cases. Surgery 152:1008-1015 